Dear author,

Please note that changes made in the online proofing system will be added to the article before publication but are not reflected in this PDF.

We also ask that this file not be used for submitting corrections. 


\section{Re: "Peroperative Intravascular Ultrasound for Endovascular Aneurysm Repair versus Peroperative Angiography: A Pilot Study in Fit Patients with Favorable Q1 Anatomy"}

\section{Dear Editor:}

It has been a pleasure reading the article titled "Peroperative Intravascular Ultrasound for Endovascular Aneurysm Repair versus Peroperative Angiography: A Pilot Study in Fit Patients with Favorable Anatomy" by Illuminati et al.

In this report, the authors describe their valuable experience in the use of intravascular ultrasound (IVUS)-assisted endovascular aortic aneurysm repair (EVAR) to address abdominal aortic aneurysms. We appreciate this well written paper and the commendable efforts of the authors but, in our opinion, some concerns arise. Despite a scare literature availability on this subject, in our opinion the authors missed to cite some recent published series ${ }^{2,3}$; in addition, we would like to highlight a few points when the IVUS is employed during EVAR procedures.

In our recent experience reported in 2019 on the same topic of IVUS EVAR and standard EVAR, ${ }^{4} 221$ patients were evaluated and 122 matched to obtain 2 homogenous groups of 26 patients each after a propensity score analysis.

The group homogeneity was assessed by analyzing 33 variables including age, sex, associated comorbidities, pre-

Q2 vious cardiac interventions, preoperative GFR $<60 \mathrm{~mL} /$ min, left ventricle ejection fraction $<50 \%$, preoperative heart function, anesthesiology classification, type of anesthesia, aneurysm behavior (maximal diameter, neck length, neck angle, neck diameter, common iliac artery involvement), and operation details (stent-graft fabric, number of components, "ballerina" configuration). The study reported a median follow-up of 27 [24 \pm 36$]$ months in the standard EVAR group and 23 [12 \pm 36$]$ months in the IVUS EVAR group.

In summary, significant differences between the 2 homogeneous groups were observed in the mean amount of contrast medium used (significantly higher in the standard EVAR group; $P=0.003$ ) and radiation exposure

\footnotetext{
Ann Vasc Surg 2020; $\mathbf{a} 1-2$

https://doi.org/10.1016/j.avsg.2020.04.045

(C) 2020 Elsevier Inc. All rights reserved.

Published online:
}

(fluoroscopy time, dose area products, and DSA runs were significantly higher in the standard EVAR group; $P=0.001,0.002$, and 0.04 respectively).

Based on the reported outcome, despite an IVUS cost increase, the IVUS EVAR was indicated in patients requiring a reduced contrast mean injection and complex cases requiring higher radiation exposure. Moreover, technical tips were recommended when dealing with IVUS EVAR including the following: (1) to acquire all IVUS images with a pull-back maneuver from the contralateral side of main body introduction; (2) to mark the lowest renal artery using short fluoroscopy spots; (3) to use the IVUS catheter-embedded radiopaque markers to measure the following distances-from the lowest renal artery to the aortic bifurcation and from the aortic bifurcation to the hypogastric arteries in both sides; and (4) to perform, in case of hostile aortic neck, a low-contrast aortography before the main body complete releasing.

Finally, from a statistical point of view, an adequate comparison between 2 different technical modalities should be performed based on homogeneous groups.

The propensity score methods represent appropriate tools despite the lack of randomization; its increased use in the recent literature, including the vascular field, is currently employed to reduce the intrinsic biases of the observational studies and to qualify the effects of the treatment outcomes. ${ }^{5}$

Felice Pecoraro

Department of Surgical

Oncological and Oral Sciences (Di.Chir.On.S.)

University of Palermo

Vascular Surgery Unit "P. Giaccone" Hospital

Palermo, Italy

Umberto Marcello Bracale

Department of Public Health

University Federico II of Naples

Vascular Surgery Unit

Naples, Italy

E-mail: felice.pecoraro@unipa.it

\section{REFERENCES}

1. Illuminati G, Pacilè MA, Ceccanei G, et al. Peroperative intravascular ultrasound for endovascular aneurysm repair versus peroperative angiography: a pilot study in fit patients with favorable anatomy. Ann Vasc Surg 2020;64:54-61.

2. Coelho A, Nogueira C, Lobo M, et al. Impact of post-EVAR graft limb kinking in EVAR limb occlusion: aetiology, early diagnosis, and management. Eur J Vasc Endovasc Surg 2019;58:681-9. 
3. Diethrich EB, Renwick B, Velineni $\mathrm{R}$, et al. Arterial and venous intravascular ultrasound applications. In: AbuRahma A ed. Noninvasive Vascular Diagnosis. Cham: Springer, 2017.

4. Pecoraro F, Bracale UM, Farina A, et al. Single-center experience and preliminary results of intravascular ultrasound in endovascular aneurysm repair. Ann Vasc Surg 2019;56: 209-15.

5. Austin PC, Cafri G. Variance estimation when using propensity-score matching with replacement with survival or time-to-event outcomes. Stat Med 2020;39: 1623-40. 